Research Article

\title{
An Electronic Architecture for Multipurpose Artificial Noses
}

\author{
Andres Gongora $\mathbb{D}$, Javier Monroy, and Javier Gonzalez-Jimenez \\ Machine Perception and Intelligent Robotics (MAPIR), University of Málaga, Málaga, Spain \\ Correspondence should be addressed to Andres Gongora; andresgongora@uma.es
}

Received 26 August 2017; Accepted 28 November 2017; Published 4 February 2018

Academic Editor: Maria Luz Rodríguez-Méndez

Copyright ( 2018 Andres Gongora et al. This is an open access article distributed under the Creative Commons Attribution License, which permits unrestricted use, distribution, and reproduction in any medium, provided the original work is properly cited.

\begin{abstract}
This paper deals with the design of an electronic device aimed at the detection and characterization of volatile chemical substances, that is, an electronic nose (e-nose). We pursue the development of a versatile, multipurpose e-nose that can be employed for a wide variety of applications, can integrate heterogeneous sensing technologies, and can offer a mechanism to be customized for different requirements. To that end, we contribute with a fully configurable and decentralized e-nose architecture based on self-contained and intelligent sensor boards (i.e., modules). This design allows for the integration not only of heterogeneous gas sensor technologies, like MOX and AEC sensors, but also of other components, such as GPS or Bluetooth, for a total of up to 127 individual modules. We describe an implementation of a fully operative prototype as an illustrative example of its potential for sensor networks, mobile robotics, and wearable technologies, each using different combinations of sensors.
\end{abstract}

\section{Introduction}

An electronic nose (e-nose) is a device aimed at the artificial perception of airborne chemical substances, a task which finds broad use in industrial applications such as environmental pollution monitoring $[1,2]$, gas source localization $[3,4]$, food quality control $[5,6]$, medical diagnosis $[7,8]$, or safety-related tasks $[9,10]$.

Typically, an e-nose detects volatile chemical substances by means of an array of nonselective gas sensors, that is, with sensors that react to a wide set of different gases, but provides no specific information about chemical identity. Therefore, the joint output of the sensor array is usually further processed by some sort of machine learning algorithm to correctly classify $[11,12]$ and/or quantify $[13,14]$ the samples. E-noses offer, as a result, a relatively cheap and fast tool to assess the presence of gases, but with a substantially greater error and uncertainty margin than precise analytic methods, like gas chromatography-mass spectrometry [15-17].

Common gas sensor technologies employed to build e-noses include metal oxide (MOX), amperometric electrochemical (AEC), quartz crystal microbalance (QCM), conducting polymers $(\mathrm{CP})$, and surface acoustic wave (SAW).
Each of these exhibit advantages and disadvantages in terms of selectivity, sensitivity, response speed, influence by environmental conditions (e.g., humidity and temperature), and drift over time among others [18-22]. But remarkably, no single technology excels in all categories.

Thus, limiting the design of an e-nose to a single sensor technology will restrict its performance and, quite often, prevent it from reaching the demanded specifications. This motivates the integration of different gas sensor technologies into a single e-nose, which would result in a sensor array with better dynamic capabilities and a more informative output [23] than any single sensor technology by its own. Since it is unfeasible to install all possible gas sensors and technologies simultaneously on a single device, it also becomes appealing to design an e-nose in such a way that its sensor array can be reconfigured depending on the applications, keeping it cost-efficient and compact.

Yet implementing these two features (heterogeneity and reconfigurability) is not simple from an electronic design perspective, as confirmed by the absence of commercial enoses fulfilling them. The former demands that very diverse and sometimes incompatible electronic characteristics be reconciled, including power requirements, noise tolerance, 
and signal processing capabilities, while the later requires a versatile mechanism to connect and remove gas sensors in a completely modular method.

To this end, we propose a novel e-nose architecture that combines self-contained and intelligent sensor boards (i.e., modules) with a decentralized design-offering a viable solution to the problem of integrating heterogeneous gas sensors in a modular fashion. This allows creating different and specific gas-sensing devices from interconnectable building blocks, which not only brings versatility and reusability to the design of e-noses but also reduces development costs and ensures long-term serviceability, as new sensors can be added as needed.

Moreover, our architecture also enables the integration of other electronic components into an e-nose, like GPS for georeferenced measurements, or wireless communications for remote readings - a feature which, despite not being a technological contribution, provides an improvement over most commercial e-noses and facilitates applications of mobile olfaction (e.g., mobile robotics or urban gas monitoring). In this regard, we must also clarify that the proposed system acts as a typical sensor, running its own firmware to provide stable and reliable measurements, but not a machine learning algorithm that depends on the final application (e.g., gas classification/quantification and odor recognition).

Overall, we believe this work to be of interest to two types of readers: those in the electronic industry, who can replicate the whole or part of our architecture to achieve a practical solution for their own gas-sensing tasks, and those within the scientific research community, who may take some insight from this work to derive new solutions toward future e-nose improvements.

The remainder of this manuscript is organized as follows. Section 2 reviews the current state of the art of e-noses, both commercial models and those proposed by other works, focusing particularly on designs that deal with the aforementioned characteristics of heterogeneity and/or reconfigurability. Afterwards, Section 3 describes the proposed e-nose architecture followed by Section 4, which describes various example prototype modules. Finally, Section 5 presents three real-use cases corresponding to specific applications, and Section 6 offers some conclusions and suggests improvements for future work.

\section{Related Work}

Due to the continuous advances in gas sensor technology, e-noses have become a viable alternative for the detection of volatile chemical substances. They not only do work in real time, allowing for fast and continuous measurements, but they are also compact enough to be carried by a person. Still, they are limited to a single gas sensor technology and lack a reconfigurable and versatile design. This section reviews some exceptions that address these limitations.

One of the first modular e-noses that hosts different chemical sensor technologies is the MOSES-II by GSG (http://www.gsg-analytical.com/english/moses2.htm). This e-nose is comprised of a base station with several MOX and QCM gas sensor modules, which can be extended with additional modules as required. However, it is conceived for precision rather than portability and is therefore too big and heavy for mobile applications.

E-noses that appeared later, like the X-am 7000 by Dräger (https://www.draeger.com/es_es/Applications/Products/ Mobile-Gas-Detection/Multi-Gas-Detection-Devices/X-am7000) or the MultiRAE by RAE Systems (http://www. raesystems.com/products/multirae), propose different solutions to combine heterogeneous gas sensors with a portable design. Concretely, these e-noses host up to 5 and 6 sensors, respectively, that can be selected from a list offered by the manufacturer. Nevertheless, they are devised and optimized for the detection of a very specific set of gases and concentration ranges (usually related to human safety at industrial plants) and have, consequently, a specific and limited number of available options.

Likewise, there are similar e-noses published in research. De Vito et al. [24] developed a prototype with ten gas sensors of different technologies, each handled by an individual electronic sublayer that accounts for specific electronic requirements. This offers a solution to the integration of heterogeneous gas sensors but does not address the problem of versatility, as the number of sensors is fixed.

In that line, we proposed in a previous work [25] a design that separates the electronics of the individual sensors and combines it with a modular approach. It comprises a main board that handles all shared resources and various smart modules that can be connected to it (following a masterslave topology), allowing to mix and replace the sensors as needed, and also to add auxiliary modules like a GPS or an SD card. However, this e-nose is still not very versatile because the specifications of the main board (e.g., I/O interfaces and power requirements) limit the scope of possible applications and because the number of sensors that this e-nose can carry is limited by the number of available slots (each module carries only one sensor). As a result, our present architecture builds on this idea of smart modules but replaces the main board with a decentralized architecture so that no module is mandatory, which means that it is possible either to assemble an e-nose with the absolute minimum required components in favour of size, weight, and cost or to upgrade it with additional sensors if needed.

In summary, this paper builds on previous research to increase the versatility of current e-noses and approaches the problem of combining multiple gas-sensing technologies within an architecture based on interconnectable modules that host the specific electronics of each sensor.

\section{Description of the Architecture}

The proposed architecture is based on the concept of modularity and comprises several freely interconnectable modules (function blocks) that host separate sensors and circuitry. Each module is designed to be self-contained and manages all its on-board resources, hiding the implementation details behind a common interface.

This interface makes it possible to handle all modules the same way regardless of their electrical characteristics, something fundamental when dealing with heterogeneous gas 


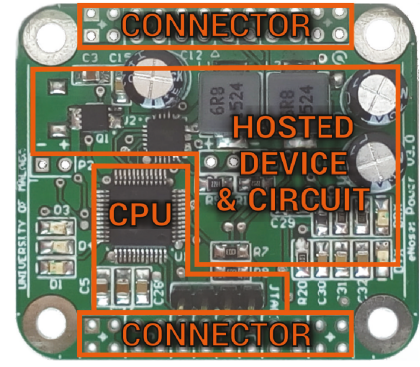

(a) Top view

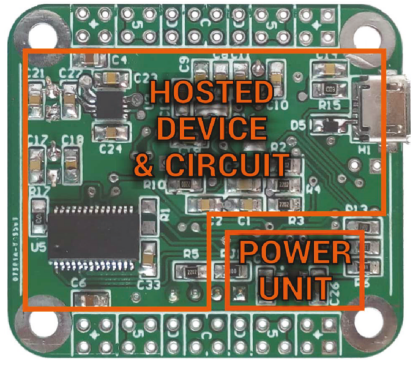

(b) Bottom view

FIGURE 1: Common components of a module (actual layout may vary depending on the implementation). The bulk of a module is comprised of the hosted device, which is conditioned by the CPU and power unit to provide a certain functionality to the e-nose. The connectors (identical on both sides) interconnect all modules to a shared power and data bus.

sensor technologies (having different electrical characteristics not only in the form of power requirements but also regarding the electrical properties associated to their transducer principles). Moreover, this versatility also enables the integration of modules with auxiliary functionalities, like data logging, wireless communications (e.g., Bluetooth, Wi-Fi, and Zigbee), or even non-gas-related sensors (e.g., GPS, humidity, and wind speed), provided that they all adhere to the same interface. Accordingly, it is also possible to design modules with several sensors in a way that is transparent to the rest of the e-nose, making it feasible to create modules with high sensor-count integrated chips [26], like those employed by Cyranose 320 (32 sensors) [27] or by Che Harun et al. (900 sensors) [28].

Modules can be connected or disconnected from the enose in a plug-and-play fashion, enabling an easy configuration based on the target application. Concretely, they are connected in a daisy chain topology that shares a single power and communications bus. Once a module is connected and powered, it works as an individual agent that handles its own resources and communicates with the others. Thus, neither a central main board is required nor is any module indispensable for the correct operation of the e-nose (power can be provided externally), allowing to assemble an e-nose with the required modules only.

Though a module is the basic element of the proposed architecture, within it, we can differentiate a series of components required for its operation (see Figure 1). These components are present in all modules, but their physical distribution and/or electronic design may vary depending on the specific implementation. In order to keep a clear and readable structure, we describe each of these components in the following subsections.

3.1. Central Processing Unit (CPU). It is the core of any module, and it is implemented as a microcontroller. It is in charge of the following tasks:

(i) Management of the particular electronics of the module: depending to the hosted circuit (sensor, communication chip, battery, etc.), the microcontroller handles polarization voltages, generation of control signals, and so forth. (ii) Data acquisition and preprocessing for sensorhosting modules: this includes, depending on the particular characteristics of the sensor, controlling the sample rates or the execution of signalconditioning operations such as noise filtering, baseline correction, or drift compensation (see [29,30] for a detailed list of signal-conditioning operations).

(iii) Communication with other modules: possibly the most important tasks of the CPU are hiding the implementation details of the hosted circuit and sharing data with other modules through a common interface. Concretely, a single physical communications bus (see Sections 3 and 3.2) is shared by all modules to communicate with each other using a broadcast service (removing the need for a central master or arbitrator and allowing each module to operate at its own sample rate).

We must stress that the microcontroller is not intended to run any sort of machine learning algorithm not only because of its limited computational resources but also because it will entail reprogramming the e-nose to adapt to the application requirements (something that contradicts the architectures' goals). Instead, the e-nose sends all the collected data to an external device, such as a computer or smartphone for further processing.

3.2. Data Power Connector. In order to enable an easy reconfiguration of the modules comprising the e-nose without the need of additional interface circuits, all modules share a common connector for power and data. More precisely, we employ two symmetrical dual-line connectors on each module. Each connector consists of a $2 \times 12$ array of $2.54 \mathrm{~mm}$-spaced pins as shown in Figure 2.

This symmetrical layout allows for lateral (Figure 3(a)), vertical (Figure 3(b)), and mixed connectivity (Figure 3(c)) of the modules in any orientation.

The pins in the connector correspond to the following: ground and $5 \mathrm{~V}$ for the power supply (see Sections 3 and 3.3) and clock and data channels for the communications bus. More specifically, we use the inter-integrated circuit $\left(\mathrm{I}^{2} \mathrm{C}\right)$ serial bus because of its practical advantages in terms of (i) availability on most microcontrollers (or the possibility 


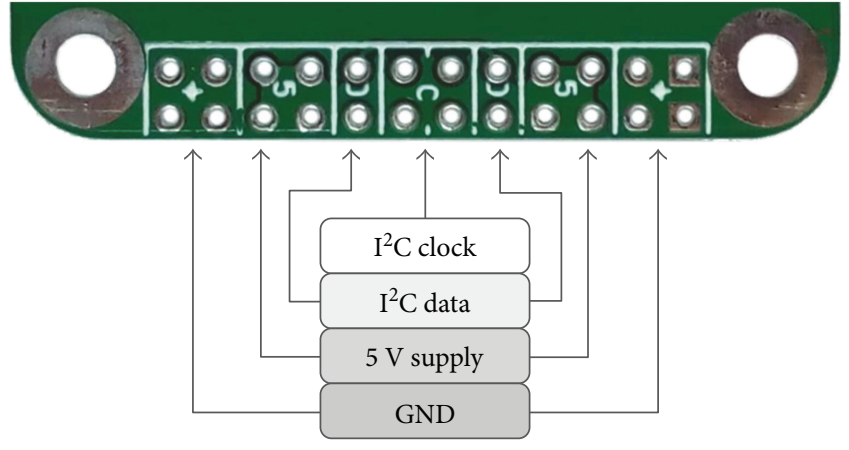

FIgURE 2: Dual-line symmetrical $2.54 \mathrm{~mm}$ spacing pinholes for the shared $5 \mathrm{~V}$ supply and for $\mathrm{I}^{2} \mathrm{C}$ communications located on both sides of a module. The symmetrical layout allows for lateral, vertical, and mixed connection in any orientation.

to be software emulated on those which it is not), (ii) support for multimaster configuration with broadcasts, (iii) handling transparently communication collisions if two modules initiate a simultaneous transmission, and (iv) use of two bidirectional open-drain lines, which favour a small connector.

As for the communication protocol, we employ MAVLink [31], a data marshaling protocol originally intended for small unmanned air vehicles, which has proven to be reliable and fast. MAVLink can be configured for the transmission of different data structures and has a built-in mechanism to identify the sender and the payload of transmitted messages, which makes it suitable for broadcast communications.

3.3. Power Unit. Most sensors and components of the e-nose require either a $5 \mathrm{~V}$ power supply (e.g., MOX gas sensors, fans, and battery management) or a $3.3 \mathrm{~V}$ power supply (microcontroller, communications, and environmental sensors). Hence, all modules are powered at $5 \mathrm{~V}$, and their power units locally reduce this voltage to $3.3 \mathrm{~V}$. This approach has the advantage of reducing complexity (modules need only one external power source), allowing the enose to be conveniently powered from any USB compliant source, despite increasing the electronic component count per module.

The power unit is also in charge of filtering the incoming $5 \mathrm{~V}$ power supply to reduce cross-talk between adjacent modules, providing separate digital and analog supplies to minimize noise coupling measurements.

3.4. Hosted Device. Finally, the last component of any module is a dedicated sensor (e.g., chemical, environmental, or physical sensor), actuator (e.g., fan and pumps), or, in general, an electronic device which provides the main functionality of the module (e.g., USB or Bluetooth communication, battery management, or data logging, among others).

\section{Prototype Modules}

Due to the versatility of the proposed architecture, no such standard prototype has been built. Instead, we implement six heterogeneous modules that can be combined into e-noses with different characteristics. Concretely, we consider two gas-sensing modules (to host different sensor technologies) and four additional modules for the management of a battery, a GPS, a data logger, and Bluetooth communication. All modules have been manufactured on a $40 \mathrm{~mm} \times 45 \mathrm{~mm}$ two-layer PCB, employing an MSP430F5309 16-bit microcontroller by Texas Instruments as the CPU.

Next, we describe these six modules, providing pictures of their implementation and detailing their electronic characteristics.

4.1. MOX Gas Sensor Module. Conductometric sensors based on metal oxide (MOX) composites are one of the most spread gas sensors, favoured due to their low price, reduced dimensions, and wide range of detectable gases [19]. And although they need to be heated to work, which incurs additional power consumption (see Table 1), controlling the temperature of MOX sensors can be used to modify their sensitivity and selectivity to certain gases [32].

Figure 4(a) shows our prototype MOX module, capable of hosting either four TO-5 Figaro sensors or two SMD MICS $S G X$ (with two transducers each). It features hardware and software low-pass filters to remove noise from sensors and supports temperature regulation by powering the sensors with individual PWM signals.

4.2. AEC Gas Sensor Module. Amperometric electrochemical (AEC) gas sensors operate by oxidizing or reducing the target gas at their electrodes, which results in a measurable current [33]. The main advantage of this technology is that it can detect very-low gas concentrations, down to a few parts per billion (ppb) in clean air, making AEC sensors a natural complement to MOX sensors (which measure parts per million).

Our AEC prototype module can only host one sensor at a time, as they are significantly bigger than MOX sensors, but supports most encapsulation formats offered by Alphasense Ltd. For example, the module shown in Figure 4(b) hosts a size B4 AEC sensor, which consumes typically $20 \mathrm{~mW}$ for continuous operation, and as listed in Table 1, up to $50 \mathrm{~mW}$ during the first warm-up hours.

Related to the specific electronics of this module, we highlight two aspects. First, AEC sensors must be polarized with a very precise voltage that depends on its electrolytic medium. We achieve this with a software-controlled closedloop PWM signal (3.3 V amplitude @ 2MHz) and a second-order RC filter (cut frequency at $500 \mathrm{~Hz}$ ) as shown in Figure 5, resulting in a programmable voltage with $1 \mathrm{mV}$ resolution between $-1.65 \mathrm{~V}$ and $+1.65 \mathrm{~V}$. Second, AEC sensors are characterized by an output signal that, ranging from a few nanoamperes to several microamperes, imposes conflicting requirements on the current voltage digitalization circuit-a high gain design can sense low gas concentrations but may saturate, while a low gain circuit suffers from reduced resolution but possesses a wider dynamic range. Instead of adhering to one option, we combine both advantages with the setup shown in Figure 5. It uses two analog amplification stages (selected by the CPU), together with a 16-bit ADS1115 ADC by Texas Instruments, so that the software can dynamically choose the best gain at any time. Concretely, the first 


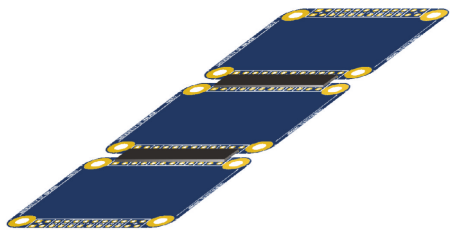

(a) Lateral connection

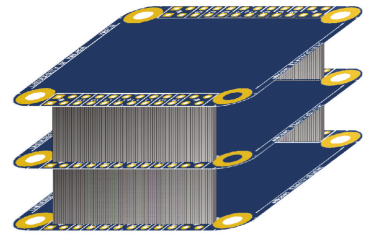

(b) Vertical connection

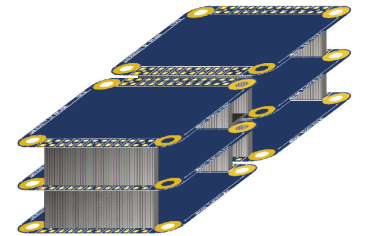

(c) Combination of lateral and vertical connection

Figure 3: Illustration of the three available configurations for connecting the modules in the e-nose, which allow for an unobstructed lateral airflow.

TABle 1: Maximum continuous power consumption of the implemented prototype modules, accounting for all components (hosted device $+\mathrm{CPU}+$ power unit), measured at $5 \mathrm{~V}$.

\begin{tabular}{lcc}
\hline $\begin{array}{l}\text { Module/ } \\
\text { component }\end{array}$ & Max. power $(\mathrm{mW})$ & Observations \\
\hline MOX (TO-5) & $4 \times 310=1240$ & 4 TO-5 sensors \\
MOX (SMD MICS) & $4 \times 100=400$ & $2 \times 2$ SMD MICS sensors \\
AEC & 50 & During the first hours \\
GPS & 150 & After acquisition \\
Bluetooth & 250 & Continuous TX \\
SD logging & 500 & Continuous logging \\
Optional fan & 1000 & For forced airflow \\
\hline
\end{tabular}

analog stage converts the sensor's current signal to voltage with a factor of $2000 \mathrm{~V} / \mathrm{A}$, the second stage amplifies it by 100 , and the ADC by an optional factor of 10 , for a total gain of either $2.000,20.000,200.000$, or 2.000.000 V/A depending on the selection.

Note that noise is mitigated by powering the analog circuitry with a dedicated TLV700 low-noise and high-PSRR voltage regulator and by various low-pass filters at each amplification stage.

4.3. Power Module. The power module (depicted in Figure $4(\mathrm{c})$ ) is intended to provide stable $5 \mathrm{~V}$ to all modules in the e-nose. It works either with an external power supply $(3.9 \mathrm{~V}$ to $15 \mathrm{~V})$, powered by USB, or with an optional onecell Li-ion rechargeable battery, with a maximum output power rating of $20 \mathrm{~W}$ and a typical efficiency of $92 \%$. Conveniently, it also serves as a USB communications module to simultaneously power and communicate with the e-nose.

The power module is built around the BQ24193 integrated battery management integrated circuit by Texas Instruments, which operates as an easy filter buck/boost switching regulator, and a FT232R USB-UART IC by FTDI Chip, which handles communications. Furthermore, it hosts passive filters to suppress noise from the $5 \mathrm{~V}$ and electronic protections against overvoltages, overcurrents, and accidental reverse-polarity connections of the external DC supply.

This module adds therefore ease-of-use, flexibility, and robustness to the e-nose, although it remains an optional module, meaning that the power module may be replaced by an external $5 \mathrm{~V}$ supply that connects directly to the common data power connector (Sections 3 and 3.2) if the application requires it.
4.4. GPS Module. One requirement of many e-nose-related applications is that the data must be georeferenced, that is, tagged with a location and time stamp. This is usually achieved with an external GPS device that accompanies the e-nose and logs its location, although it might be inconvenient in some situations. For example, it requires postprocessing to combine the e-nose and GPS data, and it is a hindrance for hand-held applications (two devices must be carried instead of one).

The proposed architecture overcomes this limitation by making it possible to add non-gas-related sensors directly to the e-nose, like in this case, a GPS. The module shown in Figure 4(d) hosts a GPS-622F board by RF Solutions Ltd. capable of obtaining the e-nose's coordinates and adding this information to other sensors. As listed in Table 1, the power consumption is less than $150 \mathrm{~mW}$, but it may reach up to $500 \mathrm{~mW}$ during initial satellite acquisition.

4.5. Memory Card Module. The memory card module (Figure 4(e)) is intended for applications that require data logging rather than live readouts over USB or Bluetooth, although it can be combined with them for more flexibility. It records the output of all sensor modules (included nongaseous sensors) on a nonvolatile secure digital (SD) card. Data is written to a binary file in the same marshaling protocol employed for the $\mathrm{I}^{2} \mathrm{C}$ bus (see Sections 3 and 3.2).

Our module features an SMD connector to hold the SD card in place, which is accessed over an SPI bus by the microcontroller. The exact power consumption depends on the write-rate and the specific SD card, but as shown in Table 1, it never exceeds $500 \mathrm{~mW}$.

4.6. Bluetooth Module. The Bluetooth module (Figure 4(f)) transparently connects the e-nose's internal $\mathrm{I}^{2} \mathrm{C}$ bus with any paired device, thus eliminating the need to connect to the e-nose over USB or simply adding a second channel to access the data. Anything written to the $\mathrm{I}^{2} \mathrm{C}$ bus is echoed over the Bluetooth link and vice versa. Because all messages are formatted following the MAVLink marshaling protocol, this communication link can be used to acquire data on a remote PC or smartphone, as well as to link two physically separated e-noses into a single device for distributed gassensing solutions.

The Bluetooth module is designed around the $\mathrm{RN}-42$ class 2 Bluetooth board by Microchips, for which its maximum power consumption lies around $250 \mathrm{~mW}$ as shown in Table 1 . This value is nevertheless much lower in practice 


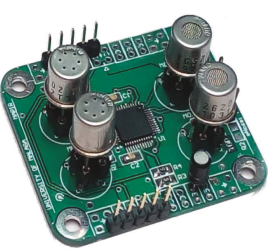

(a) MOX

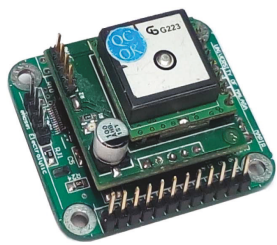

(d) GPS

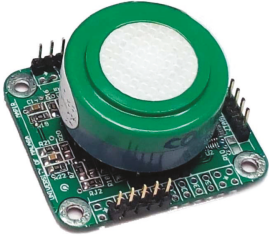

(b) Electrolytic

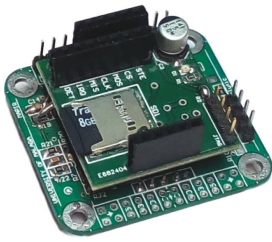

(e) SD logging

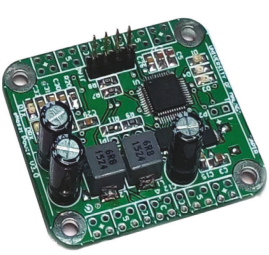

(c) Power

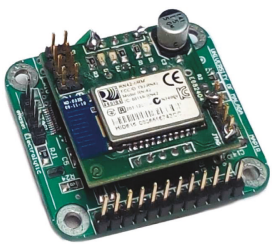

(f) Bluetooth

FIGURE 4: Six prototype modules based on the proposed architecture, hosting different sensors and electronic components, and manufactured on $40 \mathrm{~mm} \times 45 \mathrm{~mm}$ two-layer PCBs.

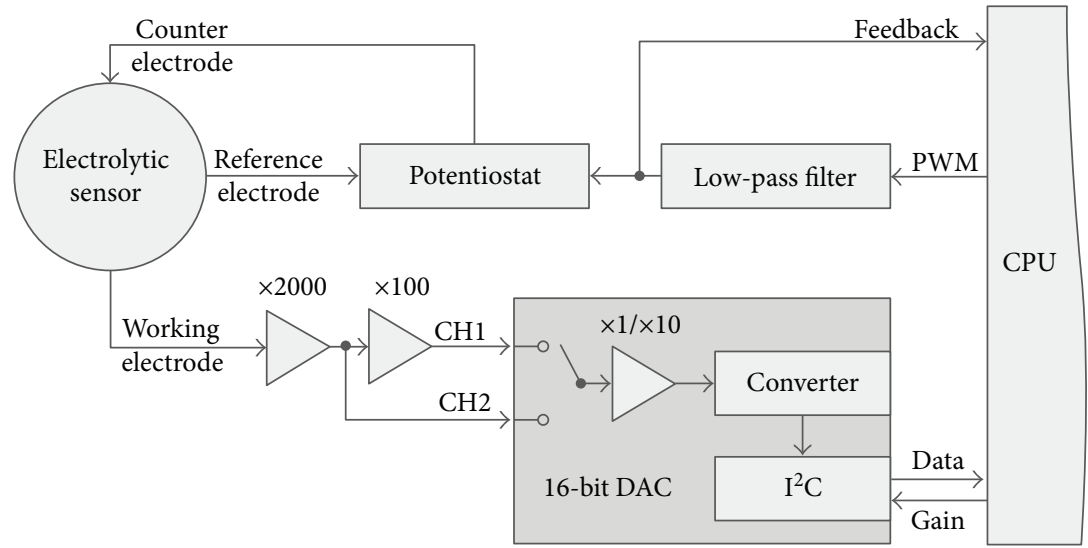

FIGURE 5: Functional block diagram of the potentiostatic circuit for amperometric electrochemical (AEC) gas sensors with variable voltage gain.

(around $100 \mathrm{~mW}$ ) and can be further decreased with a software buffer that packs various sensor readings into a single transmission.

\section{Implementation of the Case Studies}

This section illustrates how the heterogeneity and reconfigurability properties of the proposed architecture enable an easy and cost-efficient setup of different e-nose devices. Concretely, we present three real scenarios with different requirements, and, for each one, we propose a specific configuration of our e-nose. Each configuration is described by the set of interconnected modules (see Section 4), the overall electrical and physical characteristics, and by providing pictures of the resulting prototypes.

5.1. Configuration for a Monitoring Network. Applications such as air-quality monitoring, pollution control, or odor emission evaluation usually require networks of gas-sensing devices to extract the temporal and spatial information about the gases in the environment. On these networks, e-noses are installed at fixed locations; therefore, they have no special constrains regarding size and weight. The only requisites are to be sensitive to the target analytes and to be low in cost (to be installed in as many locations as possible).

For these types of applications, we propose a configuration of our e-nose based on multiple gas sensor modules (the number depends on the gases to be detected), a power module with an AC adapter that also serves for wired communications, and an enclosure equipped with a fan to accelerate the response speed of the gas sensors.

For illustrative purposes, Figure 6 shows a picture of a prototype built to be deployed in a network for the monitoring of odors released by a wastewater treatment plant [34]. For this specific application, since the only gas to monitor was sulfur hydrogen $\left(\mathrm{H}_{2} \mathrm{~S}\right)$, we only employed one electrochemical module type installed at several locations on site.

To ensure proper lateral air flow [35], the e-nose is housed in a custom $3 \mathrm{D}$-printed enclosure equipped with a fan (enclosed dimensions are $50 \mathrm{~mm}(\mathrm{~W}) \times 50 \mathrm{~mm}(\mathrm{H}) \times 55 \mathrm{~mm}$ (D)). Power consumption for the node alone is around $40 \mathrm{~mW}$ at start-up and slowly decreases (over several hours) to around $15 \mathrm{~mW}$ as the sensor reaches its working point. The fan consumes $1 \mathrm{~W}$, and the single-board computer consumes around $1.5 \mathrm{~W}$. 


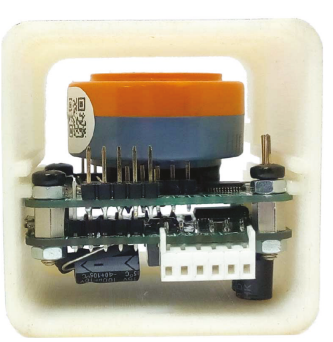

(a) Inside details

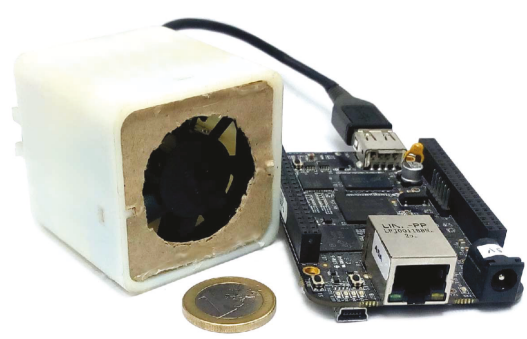

(b) Assembled node

FIGURE 6: Configuration of the e-nose to be used as part of a gas-monitoring network. (a) The power and $\mathrm{H}_{2} \mathrm{~S}$ sensor modules mounted inside the $3 \mathrm{D}$ printed enclosure (fan removed) and (b) the assembled gas-sensing node connected to a single-board computer, which provides power and communicates with the network.

5.2. Configuration for a Portable E-Nose. An alternative to the traditional sensor networks is to employ only one (or just a few) easy-to-transport e-nose. In this case, the mobile enose gathers the volatile chemical information while being transported by a robot $[36,37]$, a vehicle $[2,38]$, or a person [39], for example.

Due to the mobility constraint, the e-nose must accomplish the following specifications: (i) be sensitive to gases of interest, (ii) provide georeferenced measurements, (iii) be compact and lightweight, and (iv) either feature a data logging system or offer connectivity to an external device (e.g., computer and smartphone).

For this case scenario, we focus on an application intended to monitor the air quality in urban environments by employing portable e-noses carried by pedestrians and cyclists. The proposed prototype is shown in Figure 7, which is composed of a power module (along with a 2200 mAh lithium battery), an SD memory card module to log all measurements, a GPS module, and 4 gas-sensing modules (hosting 8 MOX sensors and 2 electrolytic sensors). The high number of gas sensors responds to the need to detect and classify multiple pollutants (classification is carried out offline in an external PC).

As in the case of the network node, the e-nose can be housed within a 3D-printed enclosure (which, following the proposed philosophy, is also modular) to allow for an easy transportation while ensuring a constant airflow through the sensors by means of a fan. We measured the power consumption to be around $2.7 \mathrm{~W}$, plus $1 \mathrm{~W}$ for the fan (total: $3.7 \mathrm{~W}$ ), yielding up to two hours of battery life. External dimensions are as follows: $50 \mathrm{~mm}(\mathrm{~W}) \times 50 \mathrm{~mm}$ (H) $\times 155 \mathrm{~mm}(\mathrm{D})$.

Finally, we tested the system by gathering data at two different locations in Málaga (Spain), where we expected to find transport emissions and potentially unpleasant odors. The first was at a very concurred sea promenade in the city center (Muelle Uno), shown in Figure 8(a), and the second was at an open-air shopping center in the outskirts (Plaza Mayor), in Figure 8(b). As expected, both maps reflect that the air is more polluted in the city center than in the outskirts. As a matter of fact, the only places at Plaza Mayor were the gas readings were consistently high was at the outdoor parking lot and around the pedestrian access to the underground parking lot.

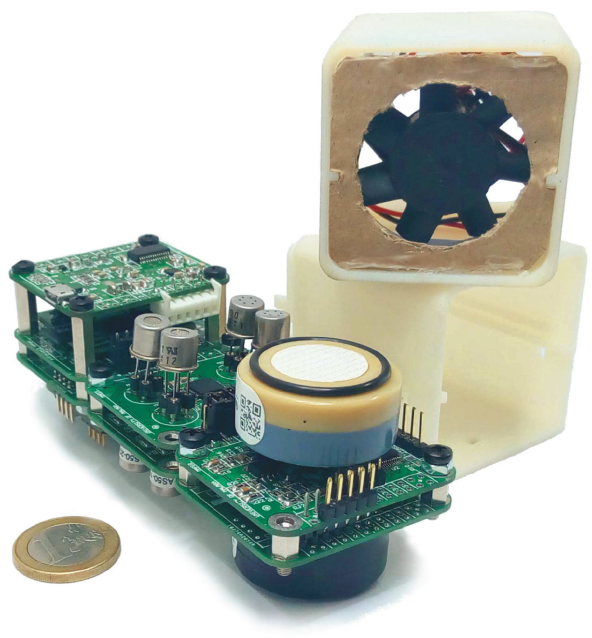

Figure 7: E-nose configured for portable operations. Each vertical stack of up to 3 modules can be hosted in a segment of a custom 3D-printed enclosure for easier handling (two segments shown, one with fan).

5.3. Configuration for a Wearable E-Nose. Wearable technology has become very popular in the last years, promoting easy-to-carry devices (either as an accessory or as part of the material used in clothing) with the ability to exchange data between the device and the network. In this line, we can set a minimal configuration of our e-nose to develop a personal air-monitoring e-nose.

The requirements of such a wearable e-nose are consequently concerned with its size, power consumption, and wireless connectivity. Here, we present a prototype consisting of only one gas sensor module (hosting a maximum of two SMD MOX sensors), a Bluetooth module (for connectivity), and a power module with a $1100 \mathrm{mAh}$ battery as shown in Figure 9. With a low Bluetooth transmission rate, the autonomy is around 28 hours with 1 SMD MOX sensor and 18 hours with 2 MOX sensors.

\section{Conclusions}

In this work, we have revised the current state of electronic noses, focusing on their ability to adapt to different requirements (target gases, concentration ranges, georeferenced measurements, and others). We have found that most 


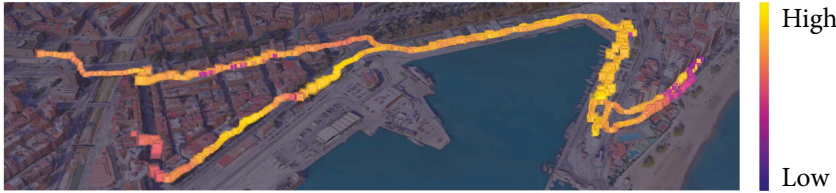

(a) Sea promenade, Muelle Uno ( $\left.36^{\circ} 43^{\prime} 4^{\prime \prime} \mathrm{N} 4^{\circ} 25^{\prime} 59^{\prime \prime} \mathrm{W}\right)$

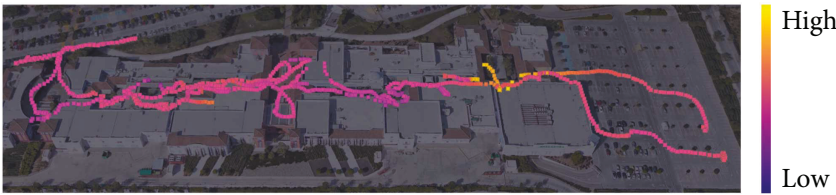

(b) Shopping center, Plaza Mayor $\left(36^{\circ} 39^{\prime} 26^{\prime \prime} \mathrm{N} 4^{\circ} 28^{\prime} 36^{\prime \prime} \mathrm{W}\right)$

FIGURE 8: Geolocalized gas concentration maps at two locations in Málaga (Spain). The displayed data correspond to the output of all sensors (averaged and normalized) of the portable e-nose configuration, targeted at air quality and pollution control in urban areas.

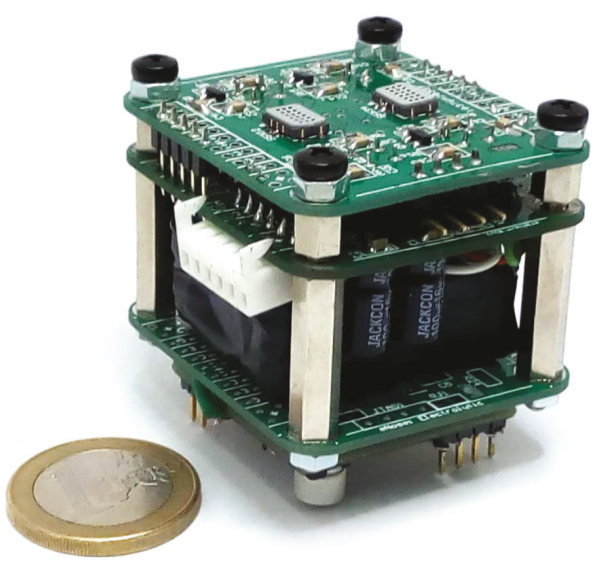

Figure 9: Wearable e-nose configuration $(45 \mathrm{~mm} \times 40 \mathrm{~mm} \times$ $40 \mathrm{~mm}$ ) intended to be paired with Bluetooth to a smartphone with up to 32 hours of autonomy. Battery can be recharged from a USB source.

e-noses are conceived for very specific applications, involving the detection of a reduced set of gases, and that they lack versatility for general-purpose artificial olfaction.

To overcome these limitations, we have proposed a novel architecture that enhances the capabilities of e-noses. It combines heterogeneous gas sensor technologies and auxiliary devices (e.g., GPS and wireless communications) in a completely modular design of interconnectable smart modules, thus enabling an easy and cost-efficient reconfiguration of the e-nose and increasing its service lifecycle, as faulty modules can be replaced individually, and new functions added when needed.

In this line, we have implemented a prototype and tested it in three real applications. Each scenario had its own and different requirements in terms of sensing capabilities, autonomy, and mobility, which were successfully met by assembling our prototype into completely different e-noses.

Finally, our next steps will focus on the development of additional modules (e.g., QCM gas sensors, ambient sensors for drift correction, and GSM communications) and on the further improvement of the functionality of our design.

\section{Conflicts of Interest}

The authors declare that they have no conflicts of interest.

\section{References}

[1] E. Bales, N. Nikzad, N. Quick, C. Ziftci, K. Patrick, and W. Griswold, "Citisense: mobile air quality sensing for individuals and communities design and deployment of the Citisense mobile air-quality system," in 2012 6th International Conference on Pervasive Computing Technologies for Healthcare (PervasiveHealth) and Workshops, pp. 155-158, San Diego, CA, USA, 2012.

[2] J. G. Monroy, J. Gonzalez-Jimenez, and C. Sanchez-Garrido, "Monitoring household garbage odors in urban areas through distribution maps," in Sensors, 2014 IEEE, pp. 1364-1367, Valencia, Spain, 2014.

[3] A. Somov, A. Baranov, D. Spirjakin, A. Spirjakin, V. Sleptsov, and R. Passerone, "Deployment and evaluation of a wireless sensor network for methane leak detection," Sensors and Actuators A: Physical, vol. 202, pp. 217-225, 2013.

[4] P. P. Neumann, V. H. Bennetts, A. J. Lilienthal, and M. Bartholmai, "From insects to micro air vehicles-a comparison of reactive plume tracking strategies," in Intelligent Autonomous Systems 13. Advances in Intelligent Systems and Computing, pp. 1533-1548, Springer, Cham, 2016.

[5] H. R. Estakhroueiyeh and E. Rashedi, "Detecting moldy bread using an e-nose and the KNN classifier," in 2015 5th International Conference on Computer and Knowledge Engineering (ICCKE), pp. 251-255, Mashhad, Iran, 2015.

[6] A. Loutfi, S. Coradeschi, G. K. Mani, P. Shankar, and J. B. B. Rayappan, "Electronic noses for food quality: a review," Journal of Food Engineering, vol. 144, pp. 103111, 2015.

[7] S. Chatterjee, M. Castro, and J.-F. Feller, "An e-nose made of carbon nanotube based quantum resistive sensors for the detection of eighteen polar/nonpolar VOC biomarkers of lung cancer," Journal of Materials Chemistry B, vol. 1, no. 36, pp. 4563-4575, 2013.

[8] M. Bruins, Z. Rahim, A. Bos, W. W. van de Sande, H. P. Endtz, and A. van Belkum, "Diagnosis of active tuberculosis by e-nose analysis of exhaled air," Tuberculosis, vol. 93, no. 2, pp. 232-238, 2013.

[9] K. Brudzewski, S. Osowski, and W. Pawlowski, "Metal oxide sensor arrays for detection of explosives at sub-parts-per million concentration levels by the differential electronic nose," Sensors and Actuators B: Chemical, vol. 161, no. 1, pp. 528-533, 2012.

[10] J. R. Askim, Z. Li, M. K. LaGasse, J. M. Rankin, and K. S. Suslick, "An optoelectronic nose for identification of explosives," Chemical Science, vol. 7, no. 1, pp. 199-206, 2016.

[11] K. Persaud and G. Dodd, "Analysis of discrimination mechanisms in the mammalian olfactory system using a model nose," Nature, vol. 299, no. 5881, pp. 352-355, 1982. 
[12] N. S. Lewis, "Comparisons between mammalian and artificial olfaction based on arrays of carbon black-polymer composite vapor detectors," Accounts of Chemical Research, vol. 37, no. 9, pp. 663-672, 2004.

[13] A. T. Guentner, V. Koren, K. Chikkadi, M. Righettoni, and S. E. Pratsinis, "E-nose sensing of low-ppb formaldehyde in gas mixtures at high relative humidity for breath screening of lung cancer?," ACS Sensors, vol. 1, no. 5, pp. 528-535, 2016.

[14] J. G. Monroy, A. J. Lilienthal, J.-L. Blanco, J. GonzalezJimenez, and M. Trincavelli, "Probabilistic gas quantification with MOX sensors in open sampling systems-a Gaussian process approach," Sensors and Actuators B: Chemical, vol. 188, pp. 298-312, 2013.

[15] S. Cui, J. Wang, L. Yang, J. Wu, and X. Wang, "Qualitative and quantitative analysis on aroma characteristics of ginseng at different ages using e-nose and GC-MS combined with chemometrics," Journal of Pharmaceutical and Biomedical Analysis, vol. 102, pp. 64-77, 2015.

[16] S.-J. Lee and A. C. Noble, "Characterization of odor-active compounds in Californian chardonnay wines using GColfactometry and GC-mass spectrometry," Journal of Agricultural and Food Chemistry, vol. 51, no. 27, pp. 8036-8044, 2003.

[17] S. Van den Velde, F. Nevens, D. van Steenberghe, and M. Quirynen, "GC-MS analysis of breath odor compounds in liver patients," Journal of Chromatography B, vol. 875, no. 2, pp. 344-348, 2008.

[18] K. J. Albert, N. S. Lewis, C. L. Schauer et al., "Cross-reactive chemical sensor arrays," Chemical Reviews, vol. 100, no. 7, pp. 2595-2626, 2000.

[19] T. C. Pearce, S. S. Schiffman, H. T. Nagle, and J. W. Gardner, Handbook of Machine Olfaction: Electronic Nose Technology, J. Wiley \& Sons, Weinheim, Germany, 2006.

[20] A. D. Wilson and M. Baietto, "Applications and advances in electronic-nose technologies," Sensors, vol. 9, no. 7, pp. 5099-5148, 2009.

[21] W. J. Buttner, M. B. Post, R. Burgess, and C. Rivkin, "An overview of hydrogen safety sensors and requirements," International Journal of Hydrogen Energy, vol. 36, no. 3, pp. 2462-2470, 2011.

[22] X. Liu, S. Cheng, H. Liu, S. Hu, D. Zhang, and H. Ning, "A survey on gas sensing technology," Sensors, vol. 12, no. 12, pp. 9635-9665, 2012.

[23] F. Rock, N. Barsan, and U. Weimar, "Electronic nose: current status and future trends," Chemical Reviews, vol. 108, no. 2, pp. 705-725, 2008.

[24] S. De Vito, E. Massera, L. Quercia, and G. Di Francia, "Analysis of volcanic gases by means of electronic nose," Sensors and Actuators B: Chemical, vol. 127, no. 1, pp. 36-41, 2007.

[25] C. Sanchez-Garrido, J. G. Monroy, and J. Gonzalez-Jimenez, "A configurable smart e-nose for spatio-temporal olfactory analysis," in Sensors, 2014 IEEE, pp. 1968-1971, Valencia, Spain, 2014.

[26] J. A. Dickson and R. M. Goodman, "Integrated chemical sensors based on carbon black and polymer films using a standard CMOS process and post-processing," in 2000 IEEE International Symposium on Circuits and Systems. Emerging Technologies for the 21st Century. Proceedings (IEEE Cat No.00CH36353), vol. 4, pp. 341-344, Geneva, Switzerland, 2000.
[27] Cyranose 320, Sensigent, http://www.sensigent.com/products/ cyranose.html.

[28] F. K. Che Harun, J. E. Taylor, J. A. Covington, and J. W. Gardner, "An electronic nose employing dual-channel odour separation columns with large chemosensor arrays for advanced odour discrimination," Sensors and Actuators, B: Chemical, vol. 141, no. 1, pp. 134-140, 2009.

[29] M. Holmberg and T. Artursson, "Drift compensation, standards, and calibration methods," in Handbook of Machine Olfaction: Electronic Nose Technology, pp. 325346, Wiley-VCH, Weinheim, Germany, 2002.

[30] K. Kashwan and M. Bhuyan, "Robust electronic-nose system with temperature and humidity drift compensation for tea and spice flavour discrimination," in 2005 Asian Conference on Sensors and the International Conference on New Techniques in Pharmaceutical and Biomedical Research, pp. 154-158, Kuala Lumpur, Malaysia, 2005.

[31] L. Meier, P. Tanskanen, F. Fraundorfer, and M. Pollefeys, "PIXHAWK: a system for autonomous flight using onboard computer vision," in 2011 IEEE International Conference on Robotics and Automation, pp. 2992-2997, Shanghai, China, 2011.

[32] A. P. Lee and B. J. Reedy, "Temperature modulation in semiconductor gas sensing," Sensors and Actuators B: Chemical, vol. 60, no. 1, pp. 35-42, 1999.

[33] S. Chang, J. Stetter, and C. Cha, "Amperometric gas sensors," Talanta, vol. 40, no. 4, pp. 461-477, 1993.

[34] C. Palacios, J. Monroy, J. Gonzalez-Jimenez, J. Olalla, and A. J. Moyano, "Monitorización y Control de la Emisión de Olores en Instalaciones de Aguas Residuales: Proyecto Sulfscan," in III Conferencia Internacional Sobres Gestión de Olores en el Medio Ambiente, Bilbao, España, http://www.olores.org.

[35] AAN 010: Design Considerations in Gas Detector SamplingAlphasense Ltd., http://www.alphasense.com/WEB1213/wpcontent/uploads/2013/07/AAN010.pdf.

[36] H. Ishida, T. Ushiku, S. Toyama, H. Taniguchi, and T. Moriizumi, "Mobile robot path planning using vision and olfaction to search for a gas source," in Sensors, 2005 IEEE, p. 4, Irvine, CA, USA, 2005.

[37] J. G. Monroy, J. L. Blanco, and J. Gonzalez-Jimenez, "Timevariant gas distribution mapping with obstacle information," Autonomous Robots, vol. 40, no. 1, pp. 1-16, 2016.

[38] D. Hasenfratz, Enabling Large-Scale Urban Air Quality Monitoring with Mobile Sensor Nodes, [Ph.D. thesis], Eidgenossische Technische Hochschule ETH Zurich, Nr. 22481, Zurich, Switzerland, 2015.

[39] A. Perera, T. Sundic, A. Pardo, R. Gutierrez-Osuna, and S. Marco, "A portable electronic nose based on embedded pc technology and GNU/Linux: hardware, software and applications," IEEE Sensors Journal, vol. 2, no. 3, pp. 235246, 2002. 


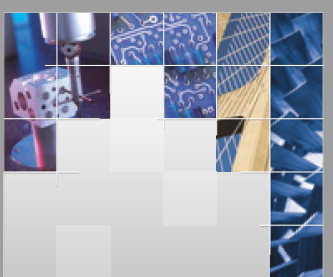

\section{Enfincering}
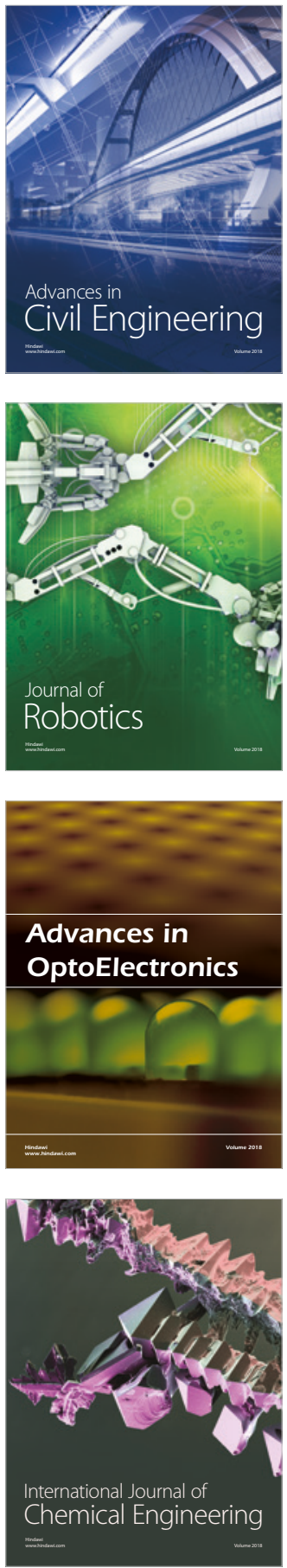

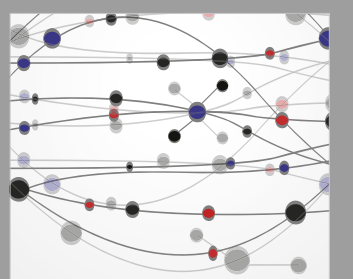

\section{Rotating \\ Machinery}

The Scientific World Journal

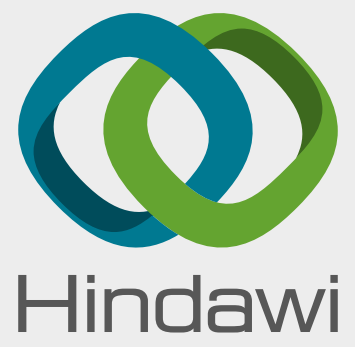

Submit your manuscripts at

www.hindawi.com
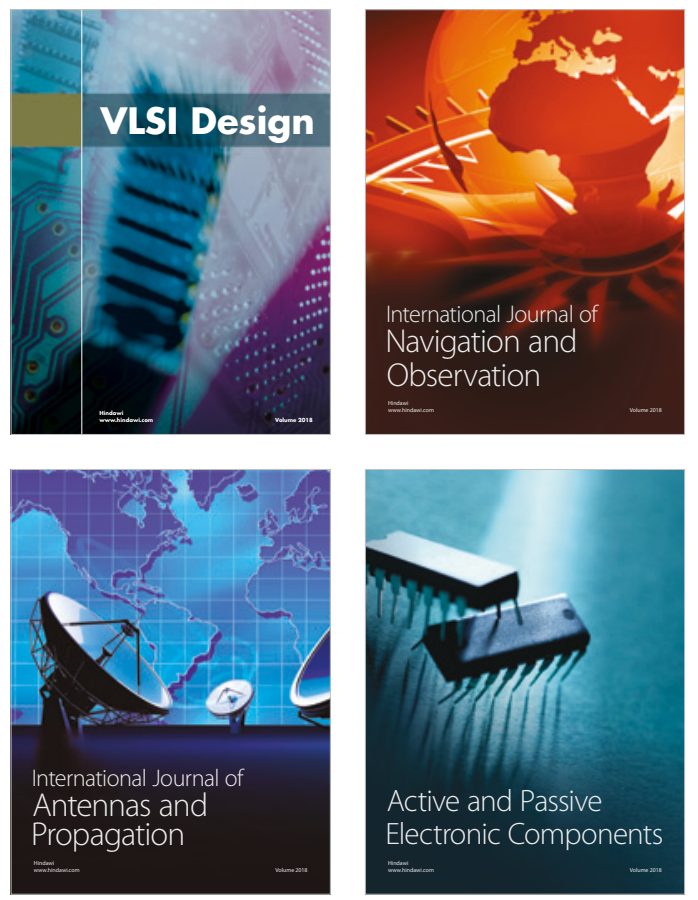
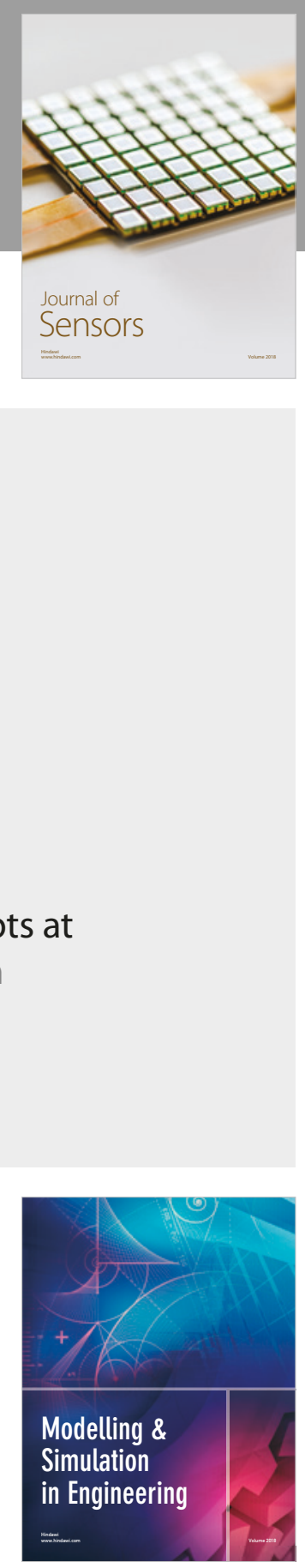

\section{Advances \\ Multimedia}
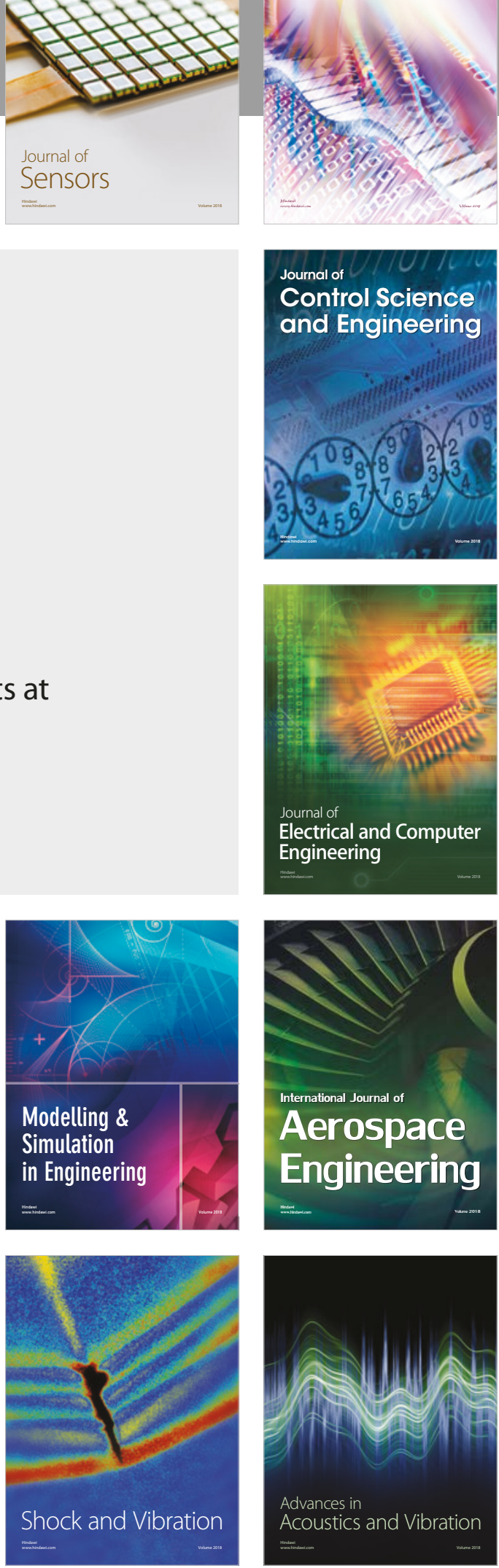